\title{
Space-Efficient Top-k Document Retrieval *
}

\author{
Gonzalo Navarro and Daniel Valenzuela \\ Dept. of Computer Science, Univ. of Chile, \{gnavarro,dvalenzu\}@dcc.uchile.cl
}

\begin{abstract}
Supporting top- $k$ document retrieval queries on general text databases, that is, finding the $k$ documents where a given pattern occurs most frequently, has become a topic of interest with practical applications. While the problem has been solved in optimal time and linear space, the actual space usage is a serious concern. In this paper we study various reduced-space structures that support top- $k$ retrieval and propose new alternatives. Our experimental results show that our novel structures and algorithms dominate almost all the space/time tradeoff.
\end{abstract}

\section{Introduction}

Ranked document retrieval is the basic task of most search engines. It consists in preprocessing a collection of $d$ documents, $\mathcal{D}=\left\{D_{1}, D_{2}, \ldots, D_{d}\right\}$, so that later, given a query pattern $P$ and a threshold $k$, one quickly finds the $k$ documents where $P$ is "most relevant".

The best known application scenario is that of documents being formed by natural language texts, that is, sequences of words, and the query patterns being words, phrases (sequences of words), or sets of words or phrases. Several relevance measures are used, which attempt to establish the significance of the query in a given document [2]. The term frequency, the number of times the pattern appears in the document, is the main component of most measures.

Ranked document retrieval is usually solved with some variant of a simple structure called an inverted index [2]. This structure, which is behind most search engines, handles well natural language collections. However, the term "natural language" hides several assumptions that are key to the efficiency of that solution: the text must be easily tokenized into words, there must not be too many different words, and queries must be whole words or phrases.

Those assumptions do not hold in various applications where document retrieval is of interest. The most obvious ones are documents written in Asian languages, where it is not easy to split words automatically, and search engines treat the text as a sequence of symbols, so that queries can retrieve any substring of the text. Other applications simply do not have a concept of word, yet ranked retrieval would be of interest: DNA or protein sequence databases where one seeks the sequences where a short marker appears frequently, source code repositories where one looks for functions making heavy use of an expression or

* Partially funded by Fondecyt Grant 1-110066, Chile. 
function call, MIDI sequence databases where one seeks for pieces where a given short passage is repeated, and so on.

These problems are modeled as a text collection where the documents $D_{i}$ are strings over an alphabet $\Sigma$, of size $\sigma$, and the queries are also simple strings. The most popular relevance measure is the term frequency, meaning the number of occurrences of the string $P$ in the strings $D_{i}$ (we discuss other measures in Section 6). We call $n=\sum\left|D_{i}\right|$ the collection size and $m=|P|$ the pattern length.

Muthukrishnan [17] pioneered the research on document retrieval for general strings. He solved the simpler problem of "document listing": reporting the occ distinct documents where $P$ appears in optimal time $O(m+o c c)$ and linear space, $O(n)$ integers (or $O(n \log n)$ bits). Muthukrishnan also considered various other document retrieval problems, but not top- $k$ retrieval.

The first efficient solution for the top- $k$ retrieval problem was introduced by Hon et al. [13]. They achieved $O(m+\log n \log \log n+k)$ time, yet the space was superlinear, $O\left(n \log ^{2} n\right)$ bits. Soon, Hon et al. [12] achieved $O(m+k \log k)$ time and linear space, $O(n \log n)$ bits. Recently, Navarro and Nekrich [18] achieved optimal time, $O(m+k)$, and reduced the space from $O(n \log n)$ to $O(n(\log \sigma+$ $\log d)$ ) bits (albeit the constant is not small).

While these solutions seem to close the problem, it turns out that the space required by $O(n \log n)$-bit solutions is way excessive for practical applications. A recent space-conscious implementation of Hon et al.'s index [20] showed that it requires at least 5 times the text size.

Motivated by this challenge, there has been a parallel research track on how to reduce the space of these solutions, while retaining efficient search time $[21,22$, $12,7,5,3,19,11]$. In this work we introduce a new variant with relevant theoretical and practical properties, and show experimentally that it dominates previous work. The next section puts our contribution in context.

\section{Related Work}

Most of the data structures for general text searching, and in particular the classical ones for document retrieval $[17,12]$, build on on suffix arrays [16] and suffix trees [23]. Regard the collection $\mathcal{D}$ as a single text $T[1, n]=D_{1} D_{2} \ldots D_{d}$, where each $D_{i}$ is terminated by a special symbol "\$". A suffix array $A[1, n]$ is a permutation of the values $[1, n]$ that points to all the suffixes of $T: A[i]$ points to the suffix $T[A[i], n]$. The suffixes are lexicographically sorted in $A: T[A[i], n]<$ $T[A[i+1], n]$ for all $1 \leq i<n$. Since the occurrences of any pattern $P$ in $T$ correspond to suffixes of $T$ that are prefixed by $P$, the occurrences are pointed from a contiguous area in the suffix array $A[s p, e p]$. A simple binary search finds $s p$ and $e p$ in $O(m \log n)$ time [16]. A suffix tree is a digital tree with $O(n)$ nodes where all the suffixes of $T$ are inserted and unary paths are compacted. Every internal node of the suffix tree corresponds to a repeated substring of $T$ and its associated suffix array interval;, suffix tree leaves correspond to the suffixes and their corresponding suffix array cells. A top-down traversal in the suffix tree finds the internal node (called the locus of $P$ ) from where all the suffixes prefixed 
with $P$ descend, in $O(m)$ time. Once $s p$ and $e p$ are known, the top- $k$ query finds the $k$ documents where most suffixes in $A[s p, e p]$ start.

A first step towards reducing the space in top- $k$ solutions is to compress the suffix array. Compressed suffix arrays (CSAs) simulate a suffix array within as little as $n H_{k}(T)+o(n \log \sigma)$ bits, for any $k \leq \alpha \log _{\sigma} n$ and any constant $0<\alpha<1$. Here $H_{k}(T)$ is the $k$-th order entropy of $T$, a measure of its statistical compressibility. The CSA, using $|C S A|$ bits, finds $s p$ and $e p$ in time $\operatorname{search}(m)$, and computes any cell $A[i]$, and even $A^{-1}[i]$, in time lookup $(n)$. For example, a CSA achieving the small space given above [6] achieves search $(m)=O(m(1+$ $\left.\left.\frac{\log \sigma}{\log \log n}\right)\right)$ and $\operatorname{lookup}(n)=O\left(\log ^{1+\epsilon} n\right)$ for any constant $\epsilon>0$. CSAs also replace the collection, as they can extract any substring of $T$.

In their very same foundational paper, Hon et al. [12] proposed an alternative succinct data structure to solve the top- $k$ problem. Building on a solution by Sadakane [21] for document listing, they use a CSA for $T$ and one smaller CSA for each document $D_{i}$, plus a little extra data, for a total space of $2|C S A|+o(n)+$ $d \log (n / d)+O(d)$ bits. They achieve time $O\left(\operatorname{search}(m)+k \log ^{3+\epsilon} n \cdot\right.$ lookup $\left.(n)\right)$, for any constant $\epsilon>0$. Gagie et al. [7] slightly reduced the time to $O(\operatorname{search}(m)+$ $\left.k \log d \log (d / k) \log ^{1+\epsilon} n \cdot \operatorname{lookup}(n)\right)$, and Belazzougui and Navarro [3] further improved it to $O\left(\operatorname{search}(m)+k \log k \log (d / k) \log ^{\epsilon} n \cdot \operatorname{lookup}(n)\right)$.

The essence of the succinct solution by Hon et al. [12] is to preprocess top$k$ answers for the lowest suffix tree nodes containing any range $A[i \cdot g, j \cdot g]$ for some sampling parameter $g$. Given the query interval $A[s p, e p]$, they find the highest preprocessed suffix tree node whose interval $\left[s p^{\prime}, e p^{\prime}\right]$ is contained in $[s p, e p]$. They show that $s p^{\prime}-s p<g$ and $e p-e p^{\prime}<g$, and then the cost of correcting the precomputed answer using the extra occurrences at $A\left[s p, s p^{\prime}-1\right]$ and $A\left[e p^{\prime}+1, e p\right]$ is bounded. For each such extra occurrence $A[i]$, one finds out its document, computes the number of occurrences of $P$ within that document, and lets the document compete in the top- $k$ precomputed list. Hon et al. use the individual CSAs and other data structures to carry out this task. The subsequent improvements $[7,3]$ are due to small optimizations on this basic design.

Gagie et al. [7] also pointed out that in fact Hon et al.'s solution can run on any other data structure able to (1) telling which document corresponds to a given $A[i]$, and (2) count how many times the same document appears in any interval $A[s p, e p]$. A structure that is suitable for this task is the document array $D[1, n]$, where $D[i]$ is the document $A[i]$ belongs to [17]. While in Hon et al.'s solution this is computed from $A[i]$ using $d \log (n / d)+O(d)$ extra bits [21], we need more machinery for task (2). A good alternative was proposed by Mäkinen and Valimäki [22] in order to reduce the space of Muthukrishnan's document listing solution [17]. The structure is a wavelet tree [10] on $D$. The wavelet tree represents $D$ using $n \log d+o(n) \log d$ bits and not only computes any $D[i]$ in $O(\log d)$ time, but it can also compute operation $\operatorname{rank}_{i}(D, j)$, which is the number of occurrences of document $i$ in $D[1, j]$, in $O(\log d)$ time too. This solves operation (2) as $\operatorname{rank}_{D[i]}(D, e p)-\operatorname{rank}_{D[i]}(D, s p-1)$. With the obvious disadvantage of the considerable extra space to represent $D$, this solution changes lookup $(n)$ by $\log d$ in the query time. Gagie et al. show many other combinations 
that solve (1) and (2). One of the fastest uses Golynski et al.'s representation [9] on $D$ and, within the same space, changes lookup $(n)$ to $\log \log d$ in the time. Very recently, Hon, Shah, and Thankachan [11] presented new combinations in the line of Gagie et al., using also faster CSAs. The least space-consuming one requires $n \log d+n o(\log d)$ bits of extra space on top of the CSA of $T$, and improves the time to $O\left(\operatorname{search}(m)+k\left(\log k+(\log \log n)^{2+\epsilon}\right)\right)$.

Belazzougui and Navarro [3] used an approach based on minimum perfect hash functions to replace the array $D$ by a weaker data structure that takes $O(n \log \log \log d)$ bits of space and supports the search in time $O(\operatorname{search}(m)+$ $\left.k \log k \log ^{1+\epsilon} n \cdot \operatorname{lookup}(n)\right)$. This is solution is intermediate between representing $D$ or the individual CSAs and it could have practical relevance.

Culpepper et al. [5] built on an improved document listing algorithm on wavelet trees [8] to achieve two top- $k$ algorithms, called Quantile and Greedy, that use the wavelet tree alone (i.e., without Hon et al.'s [12] extra structures). Despite their worst-case complexity being as bad as extracting the results one by one in $A[s p, e p]$, that is, $O((e p-s p+1) \log d)$, in practice the algorithms performed very well, being Greedy superior. They implemented Sadakane's solution [21] of using individual CSAs for the documents and showed that the overheads are very high in practice. Navarro et al. [19] arrived at the same conclusion, showing that Hon et al.'s original succinct scheme is not promising in practice: both space and time were much higher in practice than Culpepper et al.'s solution. However, their preliminary experiments [19] showed that Hon et al.'s scheme could compete when running on wavelet trees.

Navarro et al. [19] also presented the first implemented alternative to reduce the space of wavelet trees, by using Re-Pair compression [15] on the bitmaps. They showed that significant reductions in space were possible in exchange for an increase in the response time of Culpepper et al.'s Greedy algorithm (half the space and twice the time is a common figure).

This review exposes interesting contrasts between the theory and the practice in this area. On one hand, the structures that are in theory larger and faster (i.e., the $n \log d$-bits wavelet tree versus a second CSA of at most $n \log \sigma$ bits) are in practice smaller and faster. On the other hand, algorithms with no worst-case bound (Culpepper et al.'s [5]) perform very well in practice. Yet, the space of wavelet trees is still considerably large in practice (about twice the plain size of $T$ in several test collections [19]), especially if we consider that they represent totally redundant information that could be extracted from the CSA of $T$.

In this paper we study a new practical alternative. We use Hon et al.'s [12] succinct structure on top of a wavelet tree, but instead of brute force we use a variant of Culpepper et al.'s [5] method to find the extra candidate documents in $A\left[s p, s p^{\prime}-1\right]$ and $A\left[e p^{\prime}+1, e p\right]$. We can regard this combination either as Hon et al.'s method boosting Culpepper et al. or vice versa. Culpepper et al. boost Hon et al.'s method, while retaining its good worst-case complexities, as they find the extra occurrences more cleverly than by enumerating them all. Hon et al. boost plain Culpepper et al.'s method by having precomputed a large part of the range, and thus ensuring that only small intervals have to be handled. 
We consider the plain and the compressed wavelet tree representations, and the straightforward and novel representations of Hon et al.'s succinct structure. We compare these alternatives with the original Culpepper et al.'s method (on plain and compressed wavelet trees), to test the hypothesis that adding Hon et al.'s structure is worth the extra space. Similarly, we include in the comparison the basic Hon et al.'s method (with and without compressed structure) over Golynski et al.'s [9] sequence representation, to test the hypothesis that using Culpepper et al.'s method over the wavelet tree is worth compared to the brute force method over the fastest sequence representation [9]. This brute force method is also at the core of the new proposal by Hon et al. [11].

Our experiments show that our new algorithms and data structures dominate almost all the space/time tradeoff for this problem, becoming a new practical reference point.

\section{Implementing Hon et al.'s Succinct Structure}

The succinct structure of Hon et al. [12] is a sparse generalized suffix tree of $T$ (SGST; "generalized" means it indexes $d$ strings). It is obtained by cutting $A[1, n]$ into blocks of length $g$ and sampling the first and last cell of each block (recall that cells of $A$ are leaves of the suffix tree). Then all the lowest common ancestors $(l c a)$ of pairs of sampled leaves are marked, and a tree $\tau_{k}$ is formed with those (at most) $2 n / g$ marked internal nodes. The top- $k$ answer is stored for each marked node, using $O((n / g) k \log n)$ bits. This is done for $k=1,2,4, \ldots$, and parameter $g$ is of the form $g=k \cdot g^{\prime}$. The final space is $O\left(\left(n / g^{\prime}\right) \log d \log n\right)$ bits. This is made $o(n)$ by properly choosing $g^{\prime}$.

To answer top- $k$ queries, they search the CSA for $P$, to obtain the suffix range $A[s p, e p]$ of the pattern. Then they turn to the closest higher power of two of $k, k^{*}=2^{\lceil\log k\rceil}$, and let $g=k^{*} \cdot g^{\prime}$ be the corresponding $g$ value. They now find the locus of $P$ in the tree $\tau_{k^{*}}$ by descending from the root until finding the first node $v$ whose interval $\left[s p_{v}, e p_{v}\right]$ is contained in $[s p, e p]$. They have at $v$ the top- $k$ candidates for $\left[s p_{v}, e p_{v}\right]$ and have to correct the answer considering $\left[s p, s p_{v}-1\right]$ and $\left[e p_{v}+1, e p\right]$. Now we introduce two implementations of this idea.

\subsection{Sparsified Generalized Suffix Tree (SGST)}

Let us call $l_{i}=A[i]$ the $i$-th leaf. Given a value of $k$ we define $g=k \cdot g^{\prime}$, for a space/time tradeoff parameter $g^{\prime}$, and sample $n / g$ leaves $l_{1}, l_{g+1}, l_{2 g+1}, \ldots$, instead of sampling $2 n / g$ leaves as in the theoretical proposal. We mark internal SGST nodes $l c a\left(l_{1}, l_{g+1}\right), l c a\left(l_{g+1}, l_{2 g+1}\right), \ldots$ It is easy to prove that any $v=$ $l c a\left(l_{i g+1}, l_{j g+1}\right)$ is also $v=l c a\left(l_{r g+1}, l_{(r+1) g+1}\right)$ for some $r$ (precisely, $r$ is the rightmost sampled leaf descending from the child of $v$ that is ancestor of $l_{i g+1}$ ). Thus these $n / g$ SGST nodes suffice and can be computed in linear time [4].

Now we note that there is a great deal of redundancy in the $\log d$ trees $\tau_{k}$, since the nodes of $\tau_{2 k}$ are included in those of $\tau_{k}$, and the $2 k$ candidates stored in the nodes of $\tau_{2 k}$ contain those in the corresponding nodes of $\tau_{k}$. To 
factor out some of this redundancy we store only one tree $\tau$, whose nodes are the same of $\tau_{1}$, and record the class $c(v)$ of each node $v \in \tau$. This is $c(v)=$ $\max \left\{k, v \in \tau_{k}\right\}$ and can be stored in $\log \log d$ bits. Each node $v \in \tau$ stores the top-c $c(v)$ candidates corresponding to its interval, using $c(v) \log d$ bits, and their frequencies, using $c(v) \log n$ bits, plus a pointer to the table, and the interval itself, $\left[s p_{v}, e p_{v}\right]$, using $2 \log n$ bits. All the information on intervals and candidates is factored in this way, saving space. Note that the class does not necessarily decrease monotonically in a root-to-leaf path of $\tau$, thus we store all the topologies independently to allow for efficient traversal of the $\tau_{k}$ trees, for $k>1$. Apart from topology information, each node of such $\tau_{k}$ trees contains just a pointer to the corresponding node in $\tau$, using $\log |\tau|$ bits.

In our first data structure, the topology of the trees $\tau$ and $\tau_{k}$ is represented using pointers of $\log |\tau|$ and $\log \left|\tau_{k}\right|$ bits, respectively. To answer top- $k$ queries, we find the range $A[s p, e p]$ using a CSA (whose space and negligible time will not be reported because it is orthogonal to all the data structures). Now we find the locus in the appropriate tree $\tau_{k^{*}}$ top-down, binary searching the intervals $\left[s p_{v}, e p_{v}\right]$ of the children of the current node, and extracting those intervals using the pointer to $\tau$. By the properties of the sampling [12] it follows that we will traverse in this descent nodes $v \in \tau_{k^{*}}$ such that $[s p, e p] \subseteq\left[s p_{v}, e p_{v}\right]$, until reaching a node $v$ so that $\left[s p_{v}, e p_{v}\right]=\left[s p^{\prime}, e p^{\prime}\right] \subseteq[s p, e p] \subseteq\left[s p^{\prime}-g, e p^{\prime}+g\right]$ (or reaching a leaf $u \in \tau_{k}$ such that $[s p, e p] \subseteq\left[s p_{u}, e p_{u}\right]$, in which case $e p-s p+1<$ $2 g)$. This $v$ is the locus of $P$ in $\tau_{k^{*}}$, and we find it in time $O(m \log \sigma)$. This is negligible compared to the subsequent costs, as well as it is the CSA search.

\subsection{Succinct SGST}

Our second implementation uses represents the tree topologies without pointers. Although the tree operations are slightly slower than with pointers, this slowdown occurs on a less significant part of the search process, and a succinct representation allows one to reduce the sampling parameter $g$ for the same space.

Arroyuelo et al. [1] showed that, for the functionality it provides, the most promising succinct representation of trees is the so-called LOUDS [14]. It requires $2 N+o(N)$ bits of space (in practice, as little as $2.1 N$ ) to represent a tree of $N$ nodes, and it solves many operations in constant time (less than a microsecond in practice). We resort to their labeled trees [1] implementation, We encode the values $s p_{v}$ and $e p_{v}$, pointers to $\tau$ (in $\tau_{k}$ ), and pointers to the candidates in a separate array, indexed by the LOUDS rank of the node $v$, managing them as Arroyuelo et al. [1] manage labels. We use that implementation [1].

\section{A New Top- $k$ Algorithm}

We run a combination of the algorithm by Hon et al. [12] and those of Culpepper et al. [5], over a wavelet tree representation of the document array $D[1, n]$. Culpepper et al. introduce, among others, a document listing method (DFS) and a Greedy top- $k$ heuristic. We adapt these to our particular top- $k$ subproblem. 
If the search for the locus of $P$ ends at a leaf $u$ that still contains the interval $[s p, e p]$, Hon et al. simply scan $A[s p, e p]$ by brute force and accumulate frequencies. We use instead Culpepper et al.'s Greedy algorithm, which is always better than a brute-force scanning.

When, instead, the locus of $P$ is a node $v$ where $\left[s p_{v}, e p_{v}\right]=\left[s p^{\prime}, e p^{\prime}\right] \subseteq$ $[s p, e p]$, we start with the precomputed answer of the $k \leq k^{*}$ most frequent documents in $\left[s p^{\prime}, e p^{\prime}\right]$, and update it to consider the subintervals $\left[s p, s p^{\prime}-1\right]$ and $\left[e p^{\prime}+1, e p\right]$. We use the wavelet tree of $D$ to solve the following problem: Given an interval $D[l, r]$, and two subintervals $\left[l_{1}, r_{1}\right]$ and $\left[l_{2}, r_{2}\right]$, enumerate all the distinct values in $\left[l_{1}, r_{1}\right] \cup\left[l_{2}, r_{2}\right]$, and their frequencies in $[l, r]$. We propose two solutions, which generalize the heuristics proposed by Culpepper et al. [5].

\subsection{Restricted Depth-First Search (DFS)}

Let us consider a wavelet tree [10] representation of an array $D$. At the root, a bitmap $B[1, n]$ stores $B[i]=0$ if $D[i] \leq d / 2$ and $B[i]=1$ otherwise. The left child of the root is, recursively, a wavelet tree handling the subsequence of $D$ with values $D[i] \leq d / 2$, and the right child handles the subsequence of values $D[i]>d / 2$. Added over the $\log d$ levels, the wavelet tree requires $n \log d$ bits of space. With $o(n \log d)$ additional bits we answer in constant time any query $\operatorname{rank}_{0 / 1}(B, i)$ over any bitmap $B[14]$.

Note that any interval $D[i, j]$ can be projected into the left child of the root as $\left[i_{0}, j_{0}\right]=\left[\operatorname{rank}_{0}(B, i-1)+1, \operatorname{rank}_{0}(B, j)\right]$, and into its right child as $\left[i_{1}, j_{1}\right]=$ $\left[\operatorname{rank}_{1}(B, i-1)+1, \operatorname{rank}_{1}(B, j)\right]$, where $B$ is the root bitmap. Those can then be projected recursively into other wavelet tree nodes.

Our restricted DFS algorithm begins at the root of the wavelet tree and tracks down the intervals $[l, r]=[s p, e p],\left[l_{1}, r_{1}\right]=\left[s p, s p^{\prime}-1\right]$, and $\left[l_{2}, r_{2}\right]=$ $\left[e p^{\prime}+1, e p\right]$. More precisely, we count the number of zeros and ones in $B$ in ranges $\left[l_{1}, r_{1}\right] \cup\left[l_{2}, r_{2}\right]$, as well as in $[l, r]$, using a constant number of rank operations on $B$. If there are any zeros in $\left[l_{1}, r_{1}\right] \cup\left[l_{2}, r_{2}\right]$, we map all the intervals into the left child of the node and proceed recursively from this node. Similarly, if there are any ones in $\left[l_{1}, r_{1}\right] \cup\left[l_{2}, r_{2}\right]$, we continue on the right child of the node. When we reach a wavelet tree leaf we report the corresponding document, and the frequency is the length of the interval $[l, r]$ at the leaf.

When solving the problem in the context of top- $k$ retrieval, we can prune some recursive calls. If, at some node, the size of the local interval $[l, r]$ is smaller than our current $k$ th candidate to the answer, we stop exploring its subtree since it cannot contain competitive documents.

\subsection{Restricted Greedy}

Following the idea of Culpepper et al., we can not only stop the traversal when $[l, r]$ is too small, but also prioritize the traversal of the nodes by their $[l, r]$ value.

We keep a priority queue where we store the wavelet tree nodes yet to process, and their intervals $[l, r],\left[l_{1}, r_{1}\right]$, and $\left[l_{2}, r_{2}\right]$. The priority queue begins with one 
element, the root. Iteratively, we remove the element with highest $r-l+1$ value from the queue. If it is a leaf, we report it. Otherwise, we project the intervals into its left and right children, and insert each such children containing nonempty intervals $\left[l_{1}, r_{1}\right]$ or $\left[l_{2}, r_{2}\right]$ into the queue. As soon as the $r-l+1$ value of the element we extract from the queue is not larger than the $k$ th frequency known at the moment, we can stop.

\subsection{Heaps for the $k$ Most Frequent Candidates}

Our two algorithms solve the query assuming that we can easily know at each moment which is the $k$ th best candidate known up to now. We use a min-heap data structure for this purpose. It is loaded with the top- $k$ precomputed candidates corresponding to the interval $\left[s p^{\prime}, e p^{\prime}\right]$. At each point, the top of the heap gives the $k$ th known frequency in constant time. Given that the previous algorithms stop when they reach a wavelet tree node where $r-l+1$ is not larger than the $k$ th known frequency, it follows that each time the algorithms report a new candidate, that candidate is more frequent than our $k$ th known candidate. Thus we replace the top of our heap with the reported candidate and reorder the heap (which is always of size $k$, or less until we find $k$ distinct elements in $D[s p, e p]$ ). Therefore each candidate reported $\operatorname{costs} O(\log d+\log k)$ time (there are also steps that do not yield any result, but the overall bound is still $O(g(\log d+\log k)))$.

A remaining issue is that we can find again, in our DFS or Greedy traversal, a node that was in the original top- $k$ list, and thus possibly in the heap. This means that the document had been inserted with its frequency in $D\left[s p^{\prime}, e p^{\prime}\right]$, but since it appears more times in $D[s p, e p]$, we must now increase its frequency and restore the min-heap invariant. It is not hard to maintain a hash table with forward and backward pointers to the heap so that we can track their current positions and replace their values. However, for the small $k$ values used in practice (say, ten or at most hundreds), it is more practical to scan the heap for each new candidate to insert than to maintain all those pointers upon all operations.

\section{$5 \quad$ Experimental Results}

We test our implementations of Hon et al.'s succinct structure combined with a wavelet tree (as explained, the original proposal is not competitive in practice [19]). We used three test collections of different nature: ClueWiki, a $141 \mathrm{MB}$ sample of ClueWeb09, formed by 3,334 Web pages from the English Wikipedia; KGS, a $25 \mathrm{MB}$ collection of 18,838 sgf-formatted Go game records (http://www . u-go.net/gamerecords); and Proteins, a $60 \mathrm{MB}$ collection of 143,244 sequences of Human and Mouse proteins (http://www.ebi.ac.uk/swissprot).

Our tests were run on a 4-core 8-processors Intel Xeon, 2Ghz each, with 16GB RAM and $2 \mathrm{MB}$ cache. We compiled using $\mathrm{g}^{++}$with full optimization. For queries, we selected 1,000 substrings at random positions, of length 3 and 8 , and retrieved the top- $k$ documents for each, for $k=1$ and 10 . 
Choosing our best variant. Our first round of experiments compares our different implementations of SGSTs (i.e., the trees $\tau_{k}$, see Section 3) over a single implementation of wavelet tree (Alpha, choosing the best value for $\alpha$ in each case [19]). We tested a pointer-based representation of the SGST (Ptrs, the original proposal [12]), a LOUDS-based representation (LOUDS), our variant of LOUDS that stores the topologies in a unique tree $\tau$ (LIGHT), and our variant of LIGHT that does not store frequencies of the top- $k$ candidates (XLIGHT). We used sampling steps of 200 and 400 for $g^{\prime}$. For each value of $g$, we obtain a curve with various sampling steps for the rank computations on the wavelet tree bitmaps.

We also tested different algorithms to find the top- $k$ among the precomputed candidates and remaining leaves (see Section 4): Our modified greedy (Greedy), our modified depth-first-search (DFS), and the brute-force selection procedure of the original proposal [12] on top of the same wavelet tree (Select). As this is orthogonal to the data structures used, we compare these algorithms only on top of the Ptrs structure. The other structures will be tested using the best method.

Figure 1 shows the results. Method Greedy is always better than Select (up to $80 \%$ better) and DFS (up to $50 \%$ ), which confirms intuition. Using LOUDS representation instead of Ptr had almost no impact on the time. This is because time needed to find the locus is usually negligible compared with that to explore the uncovered leaves. Further costless space gains are obtained with variant LIGHT. Variant XLIGHT, instead, reduces the space of LIGHT at a noticeable cost in time that makes it not so interesting, except on Proteins. In various cases the sparser sampling dominates the denser one, whereas in others the latter makes the structure faster if sufficient space is spent. To compare with other techniques, we will use variant LIGHT on ClueWiki and KGS, and XLIGHT on Proteins, both with $g^{\prime}=400$. This combination will be called generically SSGST.

Comparison with previous work. We now compare ours with previous work. The Greedy heuristic [5] is run over different wavelet-tree representations of the document array: a plain one (WT-Plain) [5], a Re-Pair compressed one (WT-RP), and a hybrid that at each wavelet tree level chooses between plain, Re-Pair, or entropy-based compression of the bitmaps (WT-Alpha) [19]. We combine these with our best implementation of Hon et al.'s structure (suffixing the previous names with +SSGST). We also consider variant Goly+SSGST [7,11], which runs the rank-based method (Select) on top of the fastest rank-capable sequence representation of the document array (Golynski et al.'s [9], which is faster than wavelet trees for rank but does not support our more sophisticated algorithms; here we used the implementation at http://libcds.recoded.cl).

Our new structures dominate most of the space-time map. When using little space, variant WT-RP+SSGST dominates, being only ocassionally and slightly superseded by WT-RP. When using more space, WT-Alpha+SSGST takes over, and finally, with even more space, WT-Plain+SSGST becomes the best choice. Most of the exceptions arise in Proteins, which due to its incompressibility [19] makes WT-Plain+SSGST essentially the only interesting variant. The alternative Goly+SSGST is no case faster than a Greedy algorithm over plain wavelet trees (WT-Plain), and takes more space. 

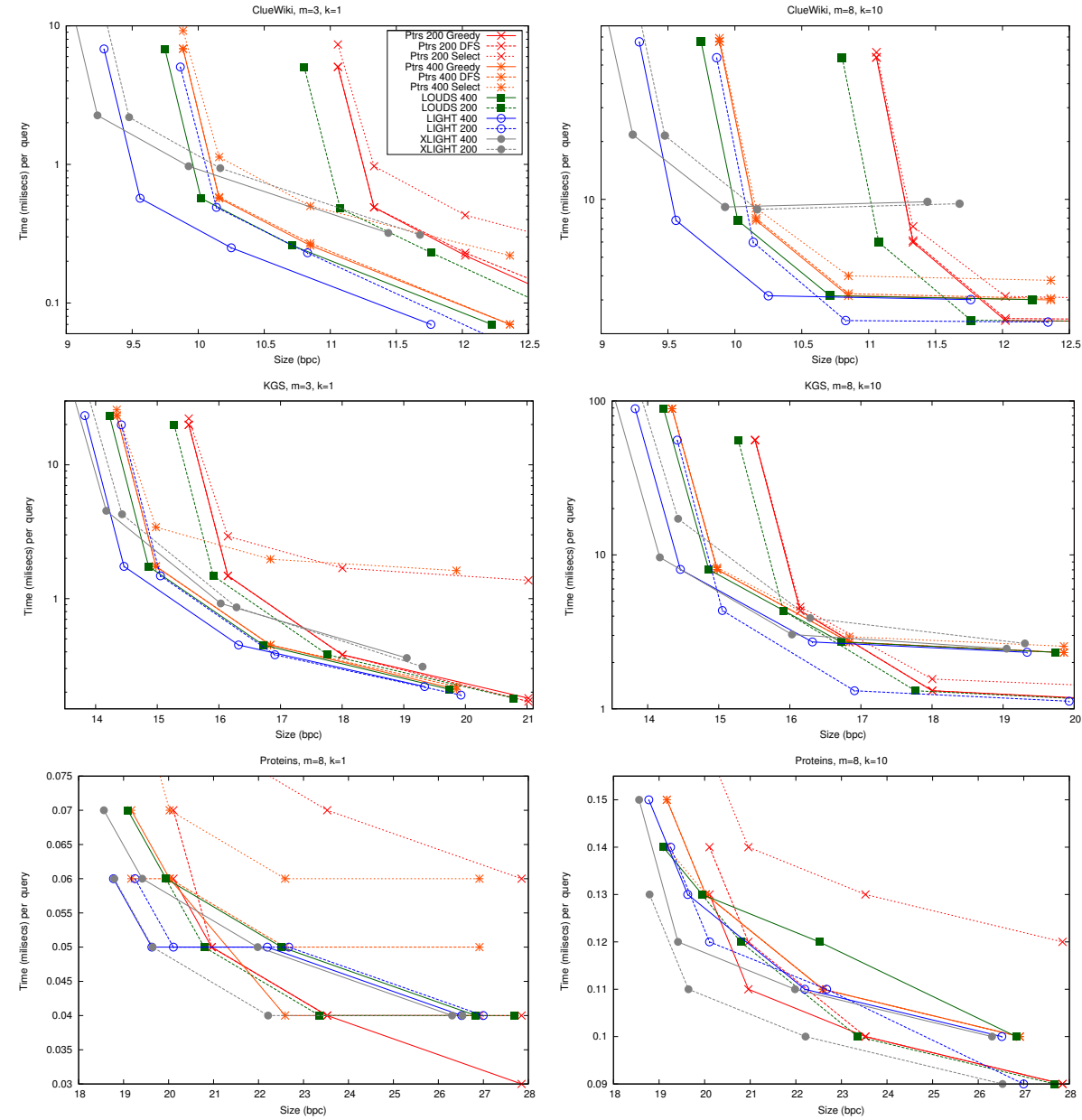

Fig. 1. Our different alternatives for top- $k$ queries. On the left for $k=1$ and pattern length $m=3$; on the right for $k=10$ and $m=8$.

\section{Final Remarks}

We can further reduce the space in exchange for possibly higher times. For example the sequence of all precomputed top- $k$ candidates can be Huffmancompressed, as there is much repetition in the sets and a zero-order compression would yield space reductions of up to $25 \%$. The pointers to those tables could also be removed, by separating the tables by size, and computing the offset within each size using rank on the sequence of classes of the nodes in $\tau$.

More in perspective, term frequency is probably the simplest relevance measure. In Information Retrieval, more sophisticated ones like BM25 are used. Such formula involves the sizes of the documents, and thus techniques like Culpep- 

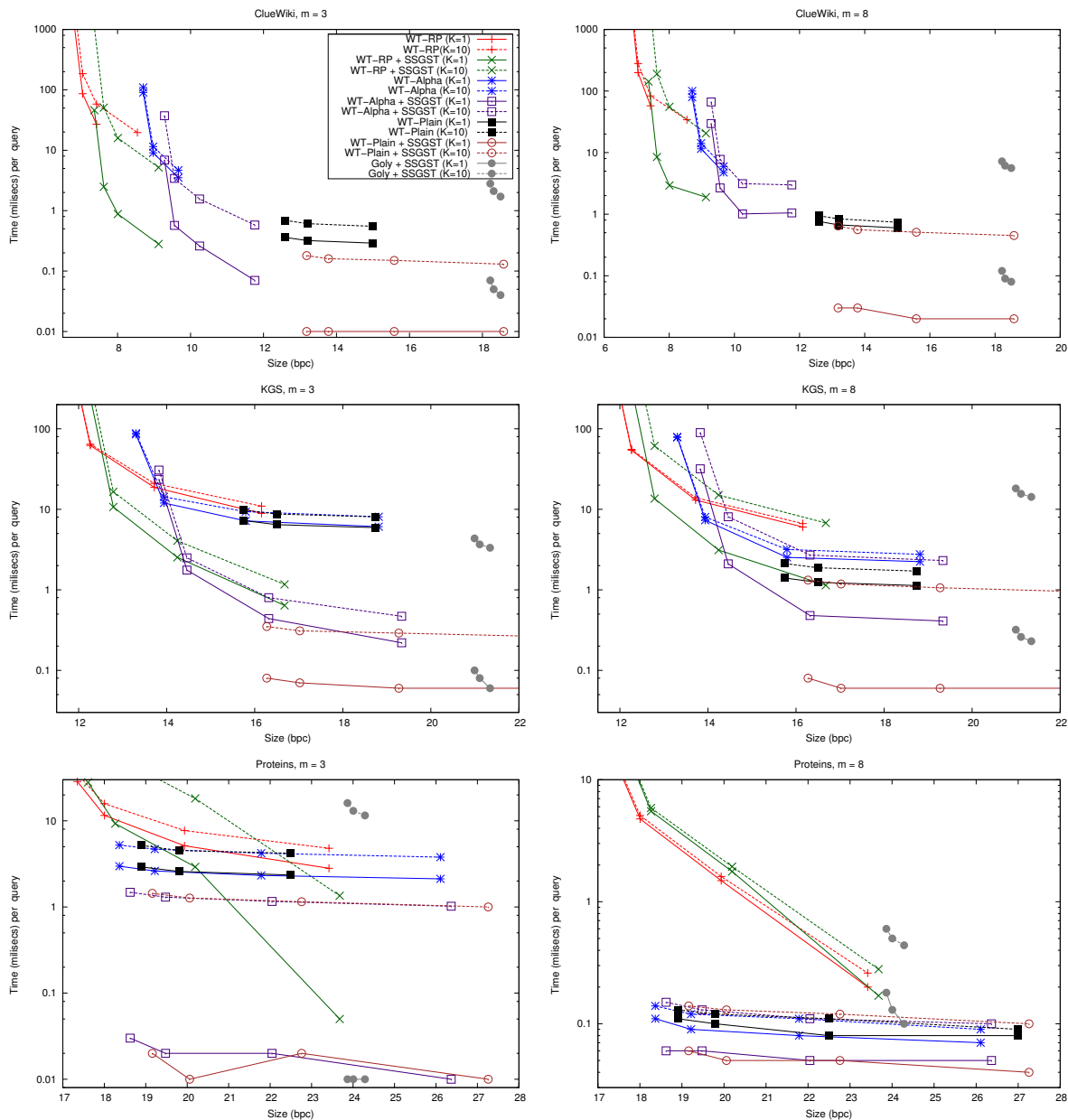

Fig. 2. Comparison with previous work, for $m=3$ (left) and $m=8$ (right).

per et al.'s [5] do not immediately apply. However, Hon et al.'s [12] does, by simply storing the precomputed top- $k$ answers according to BM25 and using their brute-force traversal instead of our "restricted Greedy/DFS" methods). The times would be very similar to the variant we called Select in this paper.

Sadakane [21] showed how to efficiently compute document frequencies (i.e., in how many documents does a pattern appear), in constant time and using just $2 n+o(n)$ bits. With term frequency, these two measures are sufficient to compute the popular tf-idf score. Note, however, that as long as queries are formed by a single term, the top- $k$ ranking is the same as given by term frequency alone. Document frequency makes a difference on bag-of-word queries, which involve several terms. Structures like those we have explored in this paper are able to emulate a (virtual) inverted list, sorted by decreasing term frequency, for any 
pattern, and thus enable the implementation of any top- $k$ algorithm for bags of words designed for inverted indexes.

\section{References}

1. D. Arroyuelo, R. Cánovas, G. Navarro, and K. Sadakane. Succinct trees in practice. In Proc. 11th ALENEX, pages 84-97, 2010.

2. R. Baeza-Yates and B. Ribeiro-Neto. Modern Information Retrieval. AddisonWesley, 2nd edition, 2011.

3. D. Belazzougui and G. Navarro. Improved compressed indexes for full-text document retrieval. In Proc. 18th SPIRE, pages 286-297, 2011.

4. M. Bender and M. Farach-Colton. The LCA problem revisited. In Proc. 2nd LATIN, pages 88-94, 2000.

5. J. S. Culpepper, G. Navarro, S. J. Puglisi, and A. Turpin. Top- $k$ ranked document search in general text databases. In Proc. 18th ESA, pages 194-205 (part II), 2010.

6. P. Ferragina, G. Manzini, V. Mäkinen, and G. Navarro. Compressed representations of sequences and full-text indexes. ACM Trans. Alg., 3(2):article 20, 2007.

7. T. Gagie, G. Navarro, and S. Puglisi. Colored range queries and document retrieval. In Proc. 17th SPIRE, pages 67-81, 2010.

8. T. Gagie, S. J. Puglisi, and A. Turpin. Range quantile queries: Another virtue of wavelet trees. In Proc. 16th SPIRE, pages 1-6, 2009.

9. A. Golynski, I. Munro, and S. Rao. Rank/select operations on large alphabets: a tool for text indexing. In Proc. 17th SODA, pages 368-373, 2006.

10. R. Grossi, A. Gupta, and J. S. Vitter. High-order entropy-compressed text indexes. In Proc. 14th SODA, pages 636-645, 2003.

11. W.-K. Hon, R. Shah, and S. Thankachan. Towards an optimal space-and-querytime index for top- $k$ document retrieval. CoRR, arXiv:1108.0554, 2011.

12. W.-K. Hon, R. Shah, and J. Vitter. Space-efficient framework for top- $k$ string retrieval problems. In Proc. 50th FOCS, pages 713-722, 2009.

13. W.-K. Hon, R. Shah, and S.-B. Wu. Efficient index for retrieving top- $k$ most frequent documents. In Proc. 16th SPIRE, pages 182-193, 2009.

14. G. Jacobson. Space-efficient static trees and graphs. In Proc. 30th FOCS, pages 549-554, 1989.

15. J. Larsson and A. Moffat. Off-line dictionary-based compression. Proc. of the IEEE, 88(11):1722-1732, 2000.

16. U. Manber and G. Myers. Suffix arrays: a new method for on-line string searches. SIAM J. Comp., 22(5):935-948, 1993.

17. S. Muthukrishnan. Efficient algorithms for document retrieval problems. In Proc. 13th SODA, pages 657-666, 2002.

18. G. Navarro and Y. Nekrich. Top- $k$ document retrieval in optimal time and linear space. In Proc. 22th SODA, pages 1066-1078, 2012.

19. G. Navarro, S. Puglisi, and D. Valenzuela. Practical compressed document retrieval. In Proc. 10th SEA, pages 193-205, 2011.

20. M. Patil, S. Thankachan, R. Shah, W.-K. Hon, J. Vitter, and S. Chandrasekaran. Inverted indexes for phrases and strings. In Proc. SIGIR, pages 555-564, 2011.

21. K. Sadakane. Succinct data structures for flexible text retrieval systems. J. Discr. Alg., 5(1):12-22, 2007.

22. N. Välimäki and V. Mäkinen. Space-efficient algorithms for document retrieval. In Proc. 18th CPM, LNCS 4580, pages 205-215, 2007.

23. P. Weiner. Linear pattern matching algorithm. In Proc. 14th Annual IEEE Symposium on Switching and Automata Theory, pages 1-11, 1973. 